\title{
Apoptosis and oxidative burst in neutrophils infected with Mycobacterium spp.
}

Carolina González-Cortés, David Reyes-Ruvalcaba, Cristina Diez-Tascón and Octavio M. Rivero-Lezcano ${ }^{1}$

Unidad de Investigación, Hospital de León, Spain

${ }^{1}$ Corresponding author at: Unidad de Investigación, Hospital of León. Bldg. S. Antonio Abad. Altos de Nava s/n, 24008-León (Spain) Tel.: +34 987234041 E-mail address: orivero@hleo.sacyl.es 


\begin{abstract}
Two of the better characterized antimicrobial mechanisms displayed by human neutrophils are the reactive oxygen species (ROS) production and the induction of apoptosis. Their importance in mycobacterial infections is, however, controversial and we aimed to analyze them simultaneously in neutrophils infected with either $M$. tuberculosis or the non-pathogenic M. gordonae. Neither species is eliminated by neutrophils but the pattern exhibited for both activities is completely different. $M$. tuberculosis induces ROS production and apoptosis but M. gordonae does not. Additional evidence was provided by an attenuated strain of $M$. gordonae that, although it has become susceptible to the antimicrobial activity of neutrophils, it still does not promote ROS production or apoptosis. Therefore no relationship could be established between any of these activities and the ability of neutrophils to kill mycobacteria. We have also observed that neutrophil concentration, a variable that is important in the antimicrobial activity against other pathogens, has no influence in the mycobacterial intracellular growth.
\end{abstract}

Keywords: M. tuberculosis; M. gordonae; innate immunity; killing; NADPH oxidase. 


\section{Introduction}

Neutrophils are fundamental cells in the innate response to infection but their importance in tuberculosis remains controversial. Several lines of evidence support the hypothesis that the innate immune response in humans is sufficient in some resistant individuals to halt or delay mycobacterial growth $[1,2]$. If this hypothesis is correct, the immune cells and mechanisms involved are unknown, but neutrophils are good candidates to participate [3].

The response of neutrophils to infection is developed through several biological activities including an oxidative burst and apoptosis. It has been assumed for a long time that the neutrophil oxidative burst, a product of the NADPH oxidase activity that generates reactive oxygen species (ROS), was associated to the neutrophil microbe killing activity. The more compelling evidence that supports this idea are the clinical symptoms exhibited by chronic granulomatous disease patients, whose phagocytes have a dysfunctional NADPH oxidase and are prone to bacterial infections. The main ROS products formed are $\mathrm{O}_{2}{ }^{-}$and $\mathrm{H}_{2} \mathrm{O}_{2}$, which may react with other ions to generate toxic compounds like hypochlorous acid [4]. A strong oxidative burst has been detected in neutrophils infected not only with $M$. tuberculosis $[5,6,7]$ but also with other mycobacteria $[8,9]$. Although it is still disputed whether neutrophils can eliminate mycobacteria, several authors that have detected a killing activity have not found it associated to the respiratory burst oxidase $[6,10]$.

Neutrophils are massively released into the blood stream and are short lived cells which undergo spontaneous apoptosis and are promptly eliminated, usually by macrophage phagocytosis [11]. Some pathogens, like Rickettsia rickettsii and Chlamidiae spp., inhibit cell death to provide a viable host cell in which to replicate and thrive. Others, like Bacillus anthracis and Bordetella pertussis induce death of immune 
cells to subvert host defence mechanisms [12]. Several investigators have shown that $M$. tuberculosis induces cell death in neutrophils [7, 13, 14, 15], but the relationship between apoptosis and antimycobacterial activity has not been analysed. It has been postulated that the phagocytosis of infected apoptotic neutrophils by macrophages induce a proinflammatory response, and that it may also be a useful mechanism of macrophage activation against mycobacteria [7, 15]. Furthermore, Perskvist et al. have found that apoptosis in M. tuberculosis infected neutrophils is oxygen-dependent [7].

Regarding the antimicrobial activity of neutrophils, early reports of in vitro infections showed that they could kill Mycobacterium tuberculosis [6, 10, 16], but these results have not been confirmed by other authors $[17,18,19]$. An important factor with a large influence in the killing activity of neutrophils, which has not been tested in mycobacteria, is the cell concentration. It has been described for other pathogens that below a critical value of $3-4 \times 10^{5}$ neutrophils $/ \mathrm{ml}$, the bacterial growth is greater than neutrophil killing [20]. In the present work we take advantage of an attenuated strain of M. gordonae, characterized in a previous report [19], which is effectively eliminated by human neutrophils. This bacterium (HL184Gat), less pigmented and easier to disperse in liquid medium, may be considered a smooth morphotype of the original rough strain (HL184G). In other non-tuberculous mycobacteria the morphological appearance of the smooth variant, which is less virulent, is influenced by the expression of a glycopeptidolipid [21]. We use both variants of M. gordonae as a comparison model for analyzing the importance of cell concentration in the elimination of mycobacteria, as well as the influence of the oxidative burst and apoptosis.

\section{Materials and methods}

\subsection{Microorganisms}


M. tuberculosis HL186T and M. gordonae HL184G were isolated at the Hospital of León (Service of Microbiology) and kindly provided by Julio Blanco and Manuela Caño. They were grown on $7 \mathrm{H} 11$ agar supplemented with $0.2 \%$ glycerol and $10 \%$ Middlebrook enrichment OADC (Becton Dickinson Microbiology Systems). After passing M. gordonae in our laboratory for more than a year we obtained an attenuated strain, M. gordonae HL184Gat [19], a smooth variant of the original rough strain (HL184G). Bacteria from fresh culture in agar plates were suspended in the serum free medium Macrophage-SFM (GIBCO). To obtain isolated mycobacteria, they were sonicated using the microtip of an S-450 digital ultrasonic cell disruptor (Branson). Pulses of $10 \mathrm{~s}$ were applied in ice with a microtip at an amplitude of $10 \%$ (2 Watts), and bacteria were centrifuged at $100 \times \mathrm{g}$ for 1 minute at room temperature. After recovering the supernatants, sonications were repeated as many times as necessary to obtain individualized bacteria, usually three or four rounds. At the end most bacteria were alive and very few groups remained, with $\leq 5$ bacteria per group, as determined by the LIVE/DEAD Baclight bacterial kit (Molecular Probes). After addition of glycerol to $20 \%$, single use aliquots were frozen at $-80^{\circ} \mathrm{C}$.

\subsection{Isolation of neutrophils}

Peripheral blood was obtained from healthy volunteers following informed consent and approval of the protocol by the Hospital of León Clinical Research Ethics Board. Each experiment was performed with blood from a different healthy volunteer. Neutrophils were isolated by dextran sedimentation and erythrocytes were removed by hypotonic lysis in cold distilled water. Mononuclear cells and neutrophils were separated by Ficoll-Paque Plus density gradient sedimentation (GE Healthcare). After removal of the supernatant, neutrophils were recovered from the bottom of the tube. The number of neutrophils was calculated by counting in a Neubauer chamber and were 
cultivated, within $3 \mathrm{~h}$ from blood collection, in the serum free medium MacrophageSFM without antibiotics (GIBCO), at $37^{\circ} \mathrm{C}$ in $95 \%$ air $/ 5 \% \mathrm{CO}_{2}$. We ascertained the purity of cells by flow cytometry. $>99 \%$ of cells were $\mathrm{CD}^{2} 6 \mathrm{~b}^{+}$(Becton Dickinson). When using an NADPH oxidase inhibitor, cells were incubated with or without $5 \mu \mathrm{M}$ diphenyleneiodonium chloride (DPI, Sigma-Aldrich).

\subsection{Neutrophil infection and antimicrobial activity}

Experiments were made in 96-well plates, always in a total volume of $100 \mu 1$. Either $5 \times 10^{4}$ or $4 \times 10^{5}$ neutrophils were infected with $10^{3}$ bacteria (multiplicity of infection, MOI $=0.02$ and 0.0025 , respectively). No washes were done at any moment. Neutrophils were lysed after $24 \mathrm{~h}$ by sonication with the microtip of an S-450 digital ultrasonic cell disruptor (Branson), at an amplitude of 10\% (2 Watts) for $3 \mathrm{~s}$, to release bacteria. At this setting ultrasounds were able to lyse cells without affecting the bacterial viability. Decimal dilutions of the sonicates were inoculated in 7H11 agar supplemented with $0.2 \%$ glycerol and 10\% Middlebrook enrichment OADC, incubated at $37^{\circ} \mathrm{C}$ and the number of colony forming units $(\mathrm{CFU})$ were counted after 8 days $(M$. gordonae) or 15 days (M. tuberculosis).

\subsection{Formation of reactive oxygen species (ROS)}

Intracellular production of ROS was measured as luminol-enhanced chemiluminiscence. $2 \times 10^{6}$ bacteria, luminol $500 \mu \mathrm{M}$ and Hanks Balanced Salt Solution were added to $4 \times 10^{5}$ neutrophils $(\mathrm{MOI}=5.0)$ in a total volume of $100 \mu \mathrm{l}$, and incubated for $30 \mathrm{~min}$ at $37^{\circ} \mathrm{C}$. Treatment of neutrophils with zymosan $(3 \mathrm{mg} / \mathrm{ml})$, which induces a strong oxidative burst, was included as a positive control. Emitted light was measured at $21^{\circ} \mathrm{C}$ at $30 \mathrm{~min}, 5 \mathrm{~h}$ and $8 \mathrm{~h}$ in a TopCount-NXT microplate scintillation and luminescence counter (Packard) and ROS formation was expressed as cps. All reagents were from Sigma-Aldrich. 


\subsection{Apoptosis quantification}

Apoptosis was detected with the Caspase-Glo 3/7 Assay kit (Promega), that provides a luminogenic substrate for caspases 3 and 7, which are activated in the last steps of apoptosis $.4 \times 10^{5}$ neutrophils were infected with $2 \times 10^{6}$ bacteria (MOI $=5.0$ ), incubated at $37^{\circ} \mathrm{C}$ for $2 \mathrm{~h}$, and lysed by ultrasounds, as described above. $2 \mu \mathrm{l}$ of cell lysate were mixed with $2 \mu \mathrm{l}$ of apoptosis luminogenic substrate. Mixtures were placed in a 96-wells white plate and after $30 \mathrm{~min}$ in the dark the protease activity was measured at $21^{\circ} \mathrm{C}$ in a TopCount-NXT microplate scintillation and luminescence counter (Packard) and expressed as counted photons per second (cps). An alternative protocol to measure apoptosis, the fluorescein FragEL DNA fragmentation kit (Calbiochem), which is based on the fluorescent labeling of DNA ends cleaved during apoptosis, was also used,. $2 \times 10^{5}$ cells were seeded on sterile $11 \mathrm{~mm}$ diameter cover glasses, infected with $1 \times 10^{6}$ bacteria, and incubated in 24 well-plates. The infection was stopped at 2,5 and $8 \mathrm{~h}$ by removal of the culture medium and fixing in $4 \%$ formaldehyde/Phosphate Buffered Saline for $15 \mathrm{~min}$. Preparations were conserved in $80 \%$ ethanol at $4^{\circ} \mathrm{C}$. Next day cells were labelled according to manufacturer's instructions, and the total number of cells was determined by DNA fluorescent staining with 4', 6-diamidino-2-phenylindole, dihydrochloride (DAPI). The proportion of apoptotic cells from DAPI stained cells was determined by fluorescent microscopy, and expressed as percentage.

\subsection{Statistical analysis}

All data, with the exception of cps (luminescence), were normally distributed (Kolmogorov-Smirnov test) with homogeneous variance (Levene test). Log transformed cps data also followed the normal distribution. Data were expressed as means and analyzed by one-way ANOVA. Pairwise comparisons of CFU were performed by the HSD Tukey's procedure. For log cps data (ROS formation and luminiscent apoptosis 
analysis) and proportion of apoptotic cells, comparisons between the control group (non-infected neutrophils, $n=8)$ and each of the neutrophil infected groups $(n=4)$ were performed by the Dunnett's t procedure. When only two groups were compared data were analyzed by Student $t$-test. In all cases, a p value $<0.05$ was considered significant. Analysis was performed with SPSS v. 14.0.

\section{Results}

\subsection{Increased neutrophil cell density does not induce antimycobacterial activity}

We have previously reported the attenuation by serial passage in our laboratory of an M. gordonae strain isolated at Hospital de León, which is efficiently eliminated by both monocytes and neutrophils at $10^{6}$ cells $/ \mathrm{ml}$, while the original M. gordonae is resistant [19]. Differences in the susceptibility to neutrophil activity may not be attributed to variations in the level of their phagocytosis. Internalization for $60 \mathrm{~min}$ of fluorescein isothiocyanate labelled bacteria (20 bacteria/neutrophil) was measured by flow cytometry, and the average number of HL184G infected cells (34.0 S.D. 13.9\%) was not significantly different from HL184Gat infected cells (37.3 S.D. $12.7 \%, \mathrm{n}=4, \mathrm{p}$ $=0.736$ by Student $t$-test).

It has been proposed that the antimicrobial activity of neutrophils depend on cell density and a critical value of $3-4 \times 10^{5}$ neutrophils $/ \mathrm{ml}$, below which the neutrophil killing activity decreases, has been defined [20]. The physiological blood neutrophil concentration is about $3-6 \times 10^{6}$ cells $/ \mathrm{ml}$, and concentrations below $5 \times 10^{5}$ cells $/ \mathrm{ml}$ are considered neutropenic. We have tested the antimicrobial activity of neutrophils against mycobacteria (M. tuberculosis and M. gordonae) with two different cell amounts: $5 \times 10^{4}$ and $4 \times 10^{5}$ cells. In our $100 \mu$ assays these quantities correspond to 5 $\times 10^{5}$ cells $/ \mathrm{ml}$, characteristic of neutropenia, and $4 \times 10^{6}$ cells $/ \mathrm{ml}$, a physiological concentration. To reduce variability we performed the infections in the absence of 
serum (Fig. 1). After $24 \mathrm{~h}$ of the infection of $5 \times 10^{4}$ neutrophils, we recovered significantly more M. tuberculosis bacteria than inoculated, implying that it was able to replicate, even at higher levels than previously reported by our group [19]. Although neutrophils did not kill M. gordonae, the bacterium did not multiply either. Finally, we have confirmed that leukocytes are able to eliminate the attenuated M. gordonae because we recovered less bacteria than inoculated $(p=0.019)$. An increased amount of neutrophils $\left(4 \times 10^{5}\right)$ did not improve, however, their antimycobacterial activity, as no significant differences in the number of surviving bacteria were found between both densities in any case. Hence, we conclude that, with the exception of M. gordonae attenuated, neutrophils do not kill mycobacteria even at high cellular density.

\subsection{Antimicrobial activity against M. gordonae attenuated does not depend on}

\section{ROS production}

It has been known for a long time that $M$. tuberculosis induces the production of ROS in neutrophils [5]. One of the current hypotheses is that activated neutrophils release ROS as a means to destroy engulfed bacteria. To verify whether the killing of $M$. gordonae attenuated was associated to ROS production we measured it in non-infected and mycobacteria infected neutrophils $(\mathrm{MOI}=5)$ for $8 \mathrm{~h}$. The antimicrobial activity at MOI $=5$ paralleled the pattern exhibited by the lower MOI described above (data not shown). As a positive control of the oxidative burst we included zymosan, which induced the generation of a large amount of ROS. When measured at $30 \mathrm{~min}$, nonopsonized M. tuberculosis induced the oxidative burst in amounts significantly different from non-infected cells $(\mathrm{p}=0.00)$. On the contrary, M. gordonae (both the original and the attenuated strains) induced a level of ROS production similar to non-infected neutrophils $(\mathrm{p}>0.8)$. This situation changed, however, when ROS was measured at 5 and $8 \mathrm{~h}$ after infection. The amount of ROS production in non-infected, zymosan 
stimulated and M. tuberculosis infected neutrophils was lower, although differences between non-infected cells and zymosan stimulated and M. tuberculosis infected cells remained significant $(\mathrm{p}=0.00)$. The behaviour of both $M$. gordonae was, however, different and the amount of ROS released remained stable at 5 and $8 \mathrm{~h}$. For this reason, the comparison with the decreased production of ROS in non-infected neutrophils became statistically significant ( $\mathrm{p} \leq 0.002)$. Therefore, killing of $M$. gordonae attenuated could not be associated with high production of ROS. Inversely, $M$. tuberculosis replicated despite the initial strong activation of the oxidative burst. Nevertheless, there might be biological implications in the way that ROS is produced in the differently infected neutrophils.

These differences in ROS production prompted us to test whether mycobacteria had any influence in the gene expression of the NADPH oxidase subunits. We infected neutrophils for $18 \mathrm{~h}$ with the mycobacteria $(\mathrm{MOI}=1)$, and analyzed by real-time PCR the level of relative gene expression of the enzymatic complex, which include the following genes: $C Y B B$ (the gene encoding the gp9 $1^{\text {phox }}$ subunit), $C Y B A\left(\mathrm{p} 22^{\text {phox }}\right), N C F 1$ (p47 $\left.7^{\text {phox }}\right), N C F 2\left(\mathrm{p} 67^{\text {phox }}\right), N C F 4\left(\mathrm{p} 40^{\text {phox }}\right)$ and $R A C 2$, being the latter the gene of a small G protein which has regulatory functions. No statistically significant differences were found in the gene expression in mycobacteria infected neutrophils as compared with non-infected cells (data not shown).

\subsection{Strains of M. gordonae do not induce apoptosis in neutrophils.}

Neutrophils undergo spontaneous apoptosis, and have a short half-life. $M$. tuberculosis also promotes cell death in neutrophils [13] and macrophages. In the latter, some hypotheses propose that the induction of apoptosis may influence mycobacterial survival [22]. We therefore wanted to know whether there was any relationship between the induction of apoptosis and the antimycobacterial activity of neutrophils. The level of 
cell death detected in non-infected neutrophils represents the spontaneous apoptosis. As expected, infection of neutrophils for $2 \mathrm{~h}$ with M. tuberculosis induced apoptosis, as measured by the use of a luminogenic substrate of caspases. Neither M. gordonae nor M. gordonae attenuated reached levels statistically significant when compared with non-infected neutrophils, although the $M$. gordonae level was higher (Table 1).

An alternative way to look at this process is the quantification of the number of apoptotic cells. The number of neutrophils which undergo apoptosis with respect to the total number of cells was determined by fluorescent microscopy with the FragEL DNA fragmentation kit at 2, 5 and 8 hours post-infection (Fig. 3). The proportion of apoptotic cells remained stable for $8 \mathrm{~h}$ in non-infected and M. gordonae infected cells, but there was a small increase in M. tuberculosis and M. gordonae attenuated infected neutrophils. Nevertheless, only M. tuberculosis induced at all times levels significantly different when compared with non-infected cells $(\mathrm{p} \leq 0.009)$. These results confirmed those obtained with the luminogenic substrate of caspases, although the levels obtained for both strains of $M$. gordonae were closer when determined by fluorescent microscopy than by luminescence. Because there were not differences in the apoptosis levels induced by both $M$. gordonae strains we conclude that apoptosis plays no role in the antimicrobial activity against $M$. gordonae attenuated.

The connection between ROS production and apoptosis in neutrophils has already been reported [Perskvist, 2002]. To test whether this relationship remains in our serum-free system we have added the NADPH oxidase inhibitor to the M. tuberculosis infection model. As expected, DPI induced a significant inhibition of ROS production (Fig. 4, panel A). Furthermore, we confirmed that DPI also decreased the level of apoptosis induced by M. tuberculosis in neutrophils (Fig. 4, panel B). We conclude 
from this experiment that ROS also induced apoptosis in our system but does not increase antimicrobial activity against $M$. tuberculosis.

\section{Discussion}

Our group is concerned about in vitro models of mycobacteria infection of human phagocytes. After passing for more than a year a $M$. gordonae strain isolated at our hospital, we observed that it had become attenuated, while we kept frozen the original strain. The attenuated strain seems to be a smooth morphotype of the rough original strain. Human phagocytes kill the attenuated strain but not the original one, which represents a useful comparative model to analyze the response of immune cells to mycobacteria infections. Our infection protocol for the analysis of antimycobacterial activity is characterized by the absence of serum and low MOI $(0.01$ bacteria/neutrophil) [19]. In the present article we take advantage of the $M$. gordonae model to test the effect of neutrophil density in its antimycobacterial activity. Li et al. showed that below a critical concentration of neutrophils, Staphylococcus epidermidis growth was greater than neutrophil killing [20]. Cell density is also important for macrophages. Boechat et al. described that a high macrophage density inhibits Mycobacterium bovis bacillus Calmette-Guérin growth [23]. Nevertheless, we have not confirmed these observations in our system, because the level of bacteria multiplication (M. tuberculosis and M. gordonae) or inhibition (M. gordonae attenuated) was not significantly different between the infection of $5 \times 10^{4}$ and $4 \times 10^{5}$ neutrophils. This result may explain why the large amount of neutrophils found in bronchoalveolar lavages from tuberculosis patients [24] is insufficient by itself to control the disease.

Regarding ROS production, it is known that is induced in neutrophils by $M$. tuberculosis [25]. We have confirmed high ROS formation (even without opsonization) and, as we had previously shown [19], resistance to neutrophil antimicrobial activity. $M$. 
tuberculosis expresses several proteins that confer resistance to ROS [26], which may explain why the oxidative burst does not impair intracellular multiplication.

Nevertheless, it has been described that other unopsonized mycobacteria, like M. phlei, M. smegmatis and M. kansasii, behave differently because they do not stimulate ROS production $[27,28]$, observations that we have also replicated for M. gordonae. Very few studies have addressed the influence of ROS in the antimycobacterial activity in neutrophils. Although Jones et al [6] have reported in an article dealing with oxidative processes the susceptibility to human neutrophils of another attenuated strain, the $M$. tuberculosis H37Ra, they did not measure the amount of ROS released in infected cells. Our model is very informative because $M$. gordonae does not induce ROS production and is not killed by neutrophils. If ROS influenced the antimicrobial activity of the cells an increased production against a susceptible strain like $M$. gordonae attenuated would be expected. Our results do not support this hypothesis, because the efficiently eliminated bacterium did not induce ROS above basal levels. Nowadays the assignation of bactericidal activity to ROS in the cell is controversial, although it has been well demonstrated in the test tube. An alternative mode of action has been proposed in which NADPH main function is to create in the long term the right conditions within the neutrophil vacuoles [29]. Even if this happens in the mycobacteria infected neutrophils, it does not seem to influence the antimicrobial activity. Furthermore, in other infection models, like Candida albicans in THP-1 cells [30], there is an increase in the transcription level of genes that codify for subunits of the NADPH oxidase complex, which may influence the intracellular environment hours after the infection. Nevertheless, we have not detected changes in the transcription of these genes in the mycobacteria infected neutrophils. 
ROS generation may induce neutrophil apoptosis [31], including when it is the result of bacterial phagocytosis [25]. Our results are consistent with this relationship because $M$. tuberculosis promotes both an oxidative burst and apoptosis. Moreover, the infection of neutrophils with M. tuberculosis in the presence of the NADPH inhibitor DPI decreases both ROS production and apoptosis. The results with both strains of $M$. gordonae are also consistent, because they do not induce either ROS production or apoptosis in infected neutrophils although $M$. gordonae seemed to induce some level of apoptosis as measured by luminescence. At this point we do not know why $M$. gordonae seems to induce some caspase activation, as measured by the luminogenic substrate. Considering that this method is very recent and has not been sufficiently characterized, we think that the determination of apoptosis by fluorescent microscopy, which does not show any difference between $M$. gordonae strains, is more reliable. Several authors suggest that apoptosis may be beneficial for the host because eliminates a pathogenic niche, thus avoiding intracellular multiplication, and permits a more efficient fusion of phagosomes and lysosomes [12]. We do not observe this phenomenon in mycobacteria infected neutrophils, because there is no relationship between apoptosis and antimicrobial activity. In fact, in a different mycobacterial model the attenuated M. tuberculosis strain H37Ra is killed by human neutrophils [16] although both H37Ra and the virulent H37Rv induce comparable levels of apoptosis [7]. Nevertheless, in this setting is difficult to affirm that apoptosis does not influence the antimicrobial activity against H37Ra. In contrast, our model provides strong evidence that this is the case. The increased susceptibility of $M$. gordonae attenuated to the neutrophil killing activity is not a consequence of neutrophil apoptosis, because for both M. gordonae strains is similar to the spontaneous level of non infected cells. 
In conclusion, we have analyzed for the first time the influence of a nontuberculous mycobacterium, M. gordonae, in ROS production and apoptosis simultaneously. Unlike M. tuberculosis, it does not induce any of these processes, although neither species is killed by neutrophils. Furthermore, an attenuated smooth variant of M. gordonae, although it has become susceptible to the neutrophils antimicrobial activity, does not promote either ROS production or apoptosis. These results provide strong support to the hypothesis that these antimicrobial mechanisms play no role against mycobacteria in neutrophils.

\section{Acknowledgments}

We thank the nurses that helped us with the blood collection. This study was supported by grants LE07/04 from Junta de Castilla y León and PI05/1288 from FIS. Dr. Rivero-Lezcano is a member of the Fundación Instituto de Estudios de Ciencias de la Salud de Castilla y León and participates in the SACYL research program. GonzálezCortés is supported by the Instituto de Salud Carlos III program for national health system research support. Reyes-Ruvalcaba is supported by Universidad Autónoma de Ciudad Juárez and fellowship UACDJ-139 from Public Education Secretary (México).

\section{References}

[1] Houk VN, Baker JH, Sorensen K, Kent DC. The epidemiology of tuberculosis infection in a closed environment. Arch Environ Health 1968; 16:26-35.

[2] Stead WW. Variation in vulnerability to tuberculosis in America today: random, or legacies of different ancestral epidemics? Int J Tuberc Lung Dis $2001 ; 5: 807-14$ 
[3] Martineau AR, Newton SM, Wilkinson KA, Kampmann B, Hall BM, Nawroly $\mathrm{N}$, et al. Neutrophil-mediated innate immune resistance to mycobacteria. J Clin Invest 2007; 117:1988-94.

[4] Roos D, van Bruggen R, Meischl C. Oxidative killing of microbes by neutrophils. Microbes Infect 2003; 5:1307-15.

[5] May ME, Spagnuolo PJ. Evidence for activation of a respiratory burst in the interaction of human neutrophils with Mycobacterium tuberculosis. Infect Immun 1987; 55:2304-07.

[6] Jones GS, Amirault HJ, Andersen BR. Killing of Mycobacterium tuberculosis by neutrophils: a nonoxidative process. J Infect Dis 1990; 162:700-4.

[7] Perskvist N, Long M, Stendahl O, Zheng L. Mycobacterium tuberculosis promotes apoptosis in human neutrophils by activating caspase- 3 and altering expression of Bax/Bcl- $x_{\mathrm{L}}$ via an oxygen-dependent pathway. J Immunol 2002; 168:6358-65.

[8] Geertsma MF, Nibbering PH, Pos O, Van Furth R. Interferon- $\gamma$-activated human granulocytes kill ingested Mycobacterium fortuitum more efficiently than normal granulocytes. Eur J Immunol 1990; 20:869-73.

[9] N'Diaye EN, Darzacq X, Astarie-Dequeker C, Daffé M, Calafat J, Maridonneau-Parini I. Fusion of azurophil granules with phagosomes and activation of the tyrosine kinase Hck are specifically inhibited during 
phagocytosis of mycobacteria by human neutrophils. J Immunol 1998; 161:4983-91.

[10] Kisich KO, Higgins M, Diamond G, Heifets L. Tumor necrosis factor alpha stimulates killing of Mycobacterium tuberculosis by human neutrophils. Infect Immun 2002; 70:4591-9.

[11] Savill JS, Wyllie AH, Henson JE, Walport MJ, Henson PM, Haslett C. Macrophage phagocytosis of aging neutrophils in inflammation. Programmed cell death in the neutrophil leads to its recognition by macrophages. J Clin Invest $1989 ; 83: 865-75$.

[12] Labbé K, Saleh M. Cell death in the host response to infection. Cell Death Differ 2008; 15:1339-49.

[13] Kasahara K, Sato I, Ogura K, Takeuchi H, Kobayashi K, Adachi M. Expression of chemokines and induction of rapid cell death in human blood neutrophils by Mycobacterium tuberculosis. J Infect Dis 1998; 178:127-137.

[14] Alemán M, Schierloh P, de la Barrera SS, Musella RM, Saab MA, Baldini M, et al. Mycobacterium tuberculosis triggers apoptosis in peripheral neutrophils involving toll-like receptor 2 and p38 mitogen protein kinase in tuberculosis patients. Infect Immun 2004; 72:5150-8.

[15] Persson YA, Blomgran-Julinder R, Rahman S, Zheng L, Stendahl O. Mycobacterium tuberculosis-induced apoptotic neutrophils trigger a pro- 
inflammatory response in macrophages through release of heat shock protein 72, acting in synergy with the bacteria. Microbes Infect 2008; 10:233-40.

[16] Majeed M, Perskvist N, Ernst JD, Orselius K, Stendahl O. Roles of calcium and annexins in phagocytosis and elimination of an attenuated strain of Mycobacterium tuberculosis in human neutrophils. Microb Pathog 1998; 24:309-20.

[17] Denis M. Human neutrophils, activated with cytokines or not, do not kill virulent Mycobacterium tuberculosis. J Infect Dis 1991; 163:919-20.

[18] Aston C, Rom WN, Talbot AT, Reibman J. Early inhibition of mycobacterial growth by human alveolar macrophages is not due to nitric oxide. Am J Respir Crit Care Med 1998; 157:1943-50.

[19] Reyes-Ruvalcaba D, González-Cortés C, Rivero-Lezcano OM. Human phagocytes lack the ability to kill Mycobacterium gordonae, a non-pathogenic mycobacteria. Immunol Lett 2008; 116:72-8.

[20] Li Y, Karlin A, Loike JD, Silverstein SC. A critical concentration of neutrophils is required for effective bacterial killing in suspension. Proc Natl Acad Sci USA 2002; 99:8289-94.

[21] Howard ST, Rhoades E., Recht J, Pang X, Alsup A, Kolter R et al. Spontaneous reversion of Mycobacterium abscessus from a smooth to a rough 
morphotype is associated with reduced expression of glycopeptidolipid and reacquisition of an invasive phenotype. Microbiology 2006; 152: 1581-90.

[22] Bocchino M, Galati D, Sanduzzi A, Colizzi V, Brunetti E, Mancino G. Role of mycobacteria-induced monocyte/macrophage apoptosis in the pathogenesis of human tuberculosis. Int J Tuberc Lung Dis 2005; 9:375-83.

[23] Boechat N, Bouchonnet F, Bonay M, Grodet A, Pelicic V, Gicquel B, et al. Culture at high density improves the ability of human macrophages to control mycobacterial growth. J Immunol 2001; 166:6203-11.

[24] Condos R, Rom WN, Liu YM, Schluger NW. Local immune responses correlate with presentation and outcome in tuberculosis. Am J Respir Crit Care Med 1998; 157:729-35.

[25] Zhang B, Hirahashi J, Cullere X, Mayadas TN. Elucidation of molecular events leading to neutrophil apoptosis following phagocytosis: cross-talk between caspase 8 , reactive oxygen species, and MAPK/ERK activation. J Biol Chem 2003; 278:28443-54.

[26] Akhtar P, Srivastava S, Srivastava A, Srivastava M, Srivastava BS, Srivastava R. Rv3303c of Mycobacterium tuberculosis protects tubercle bacilli against oxidative stress in vivo and contributes to virulence in mice. Microbes Infect $2006 ; 8: 2855-62$. 
[27] Astarie-Dequeker C, N'Diaye EN, Le Cabec V, Rittig MG, Prandi J, Maridonneau-Parini I. The mannose receptor mediates uptake of pathogenic and nonpathogenic mycobacteria and bypasses bactericidal responses in human macrophages. Infect Immun 1999; 67:469-77.

[28] Le Cabec V, Cols C, Maridonneau-Parini I. Nonopsonic phagocytosis of zymosan and Mycobacterium kansasii by CR3 (CD11b/CD18) involves distinct molecular determinants and is or is not coupled with NADPH oxidase activation. Infect Immun 2000; 68:4736-45.

[29] Segal AW. How neutrophils kill microbes. Annu Rev Immunol 2005; 23:197223.

[30] Barker KS, Liu T, Rogers PD. Coculture of THP-1 human mononuclear cells with Candida albicans results in pronounced changes in host gene expression. J Infect Dis 2005; 192:901-12.

[31] Melley DD, Evans TW, Quinlan GJ. Redox regulation of neutrophil apoptosis and the systemic inflammatory response syndrome. Clin Sci (Lond) 2005; $108: 413-24$. 


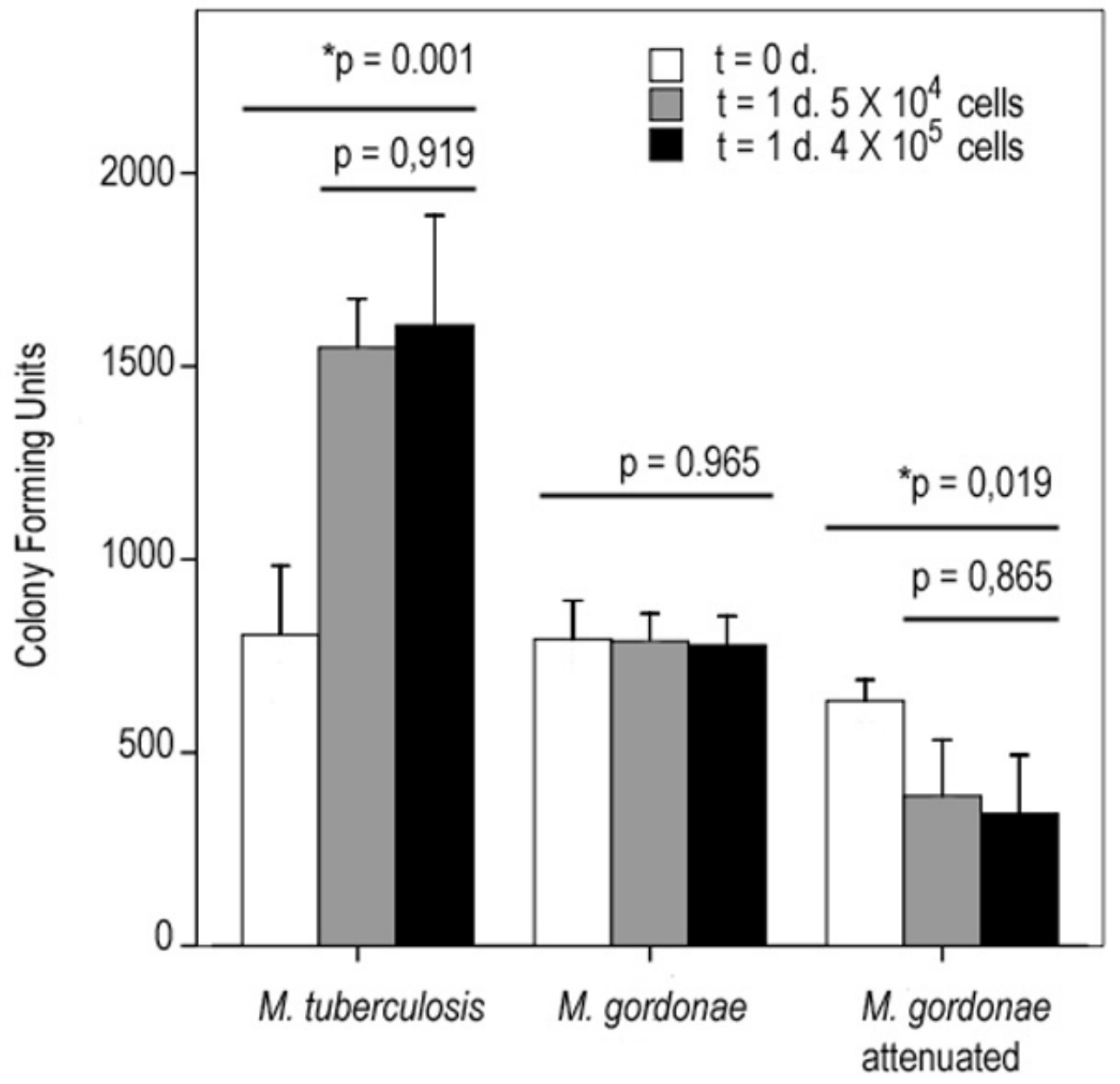

Figure 1. Antimicrobial activiy of neutrophils at different cell density. CFU at the moment of infection $(t=0 d)$ and at the time of cellular lysis $(t=1 d)$ of either $5 \times$ $10^{4}$ or $4 \times 10^{5}$ neutrophils. Cells were infected with approximately $10^{3}$ bacteria and data represent the mean + S.D. of the number of CFU of four independent experiments. The long lines correspond to the ANOVA test $\mathrm{p}$ value and the short lines to the HSD Tukey's test $p$ value. A * $p<0.05$ was considered significant. 


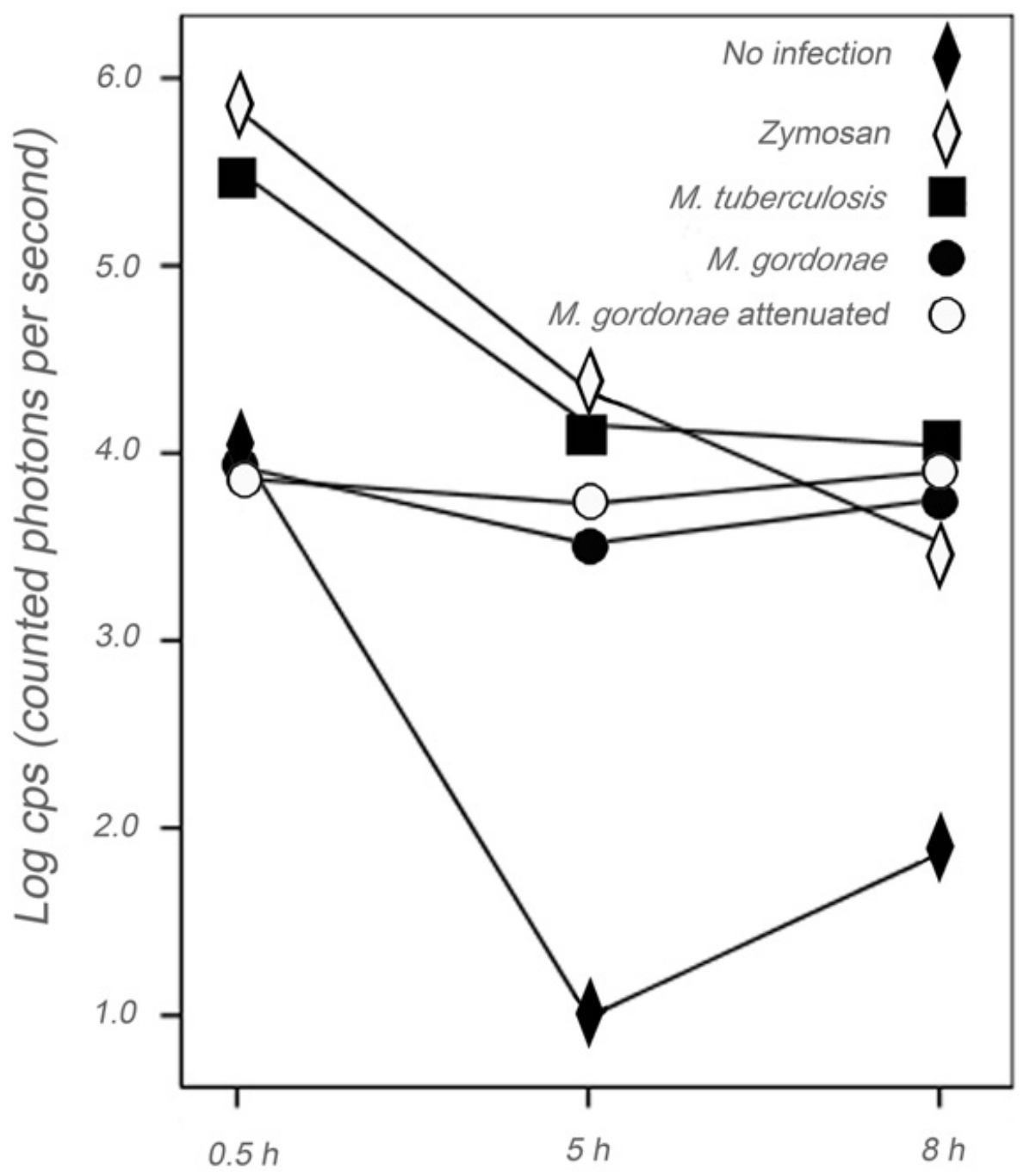

Figure 2. ROS production in infected neutrophils. $4 \times 10^{5}$ neutrophils were stimulated with zymosan or infected with $2 \times 10^{6}$ bacteria (M. tuberculosis, $M$. gordonae or M. gordonae attenuated). Non infected cells were used as control. Data are the log of counted photons per second emitted in the presence of luminol and represent the mean of four independent experiments. Measures were taken at $30 \mathrm{~min}, 5 \mathrm{~h}$ and $8 \mathrm{~h}$. 
Table 1

Apoptosis in mycobacteria infected neutrophils.

\begin{tabular}{llc}
\hline & \multicolumn{1}{c}{ Apoptosis } & $\mathrm{p}$ \\
\hline Non infected & 4.79 S.D. $0.25(4.58-5.00)$ & \\
M. tuberculosis & 5.33 S.D. $0.45(4.61-6.05)^{*}$ & 0.040 \\
M. gordonae & 5.26 S.D. $0.30(4.78-5.74)$ & 0.080 \\
M. gordonae attenuated & 4.73 S.D. $0.35(4.17-5.28)$ & 0.985 \\
\hline
\end{tabular}

$4 \times 10^{5}$ neutrophils were infected with a MOI of 5 , and a negative control (no infection) was included. Quantities represent the log of cps with standard deviation S.D. (95\% confidence interval). Dunnett's $t$ test was used for comparisons between the negative control $(n=8)$ and each of the other groups $(n=4) .{ }^{*} p<0.05$ is considered significant 


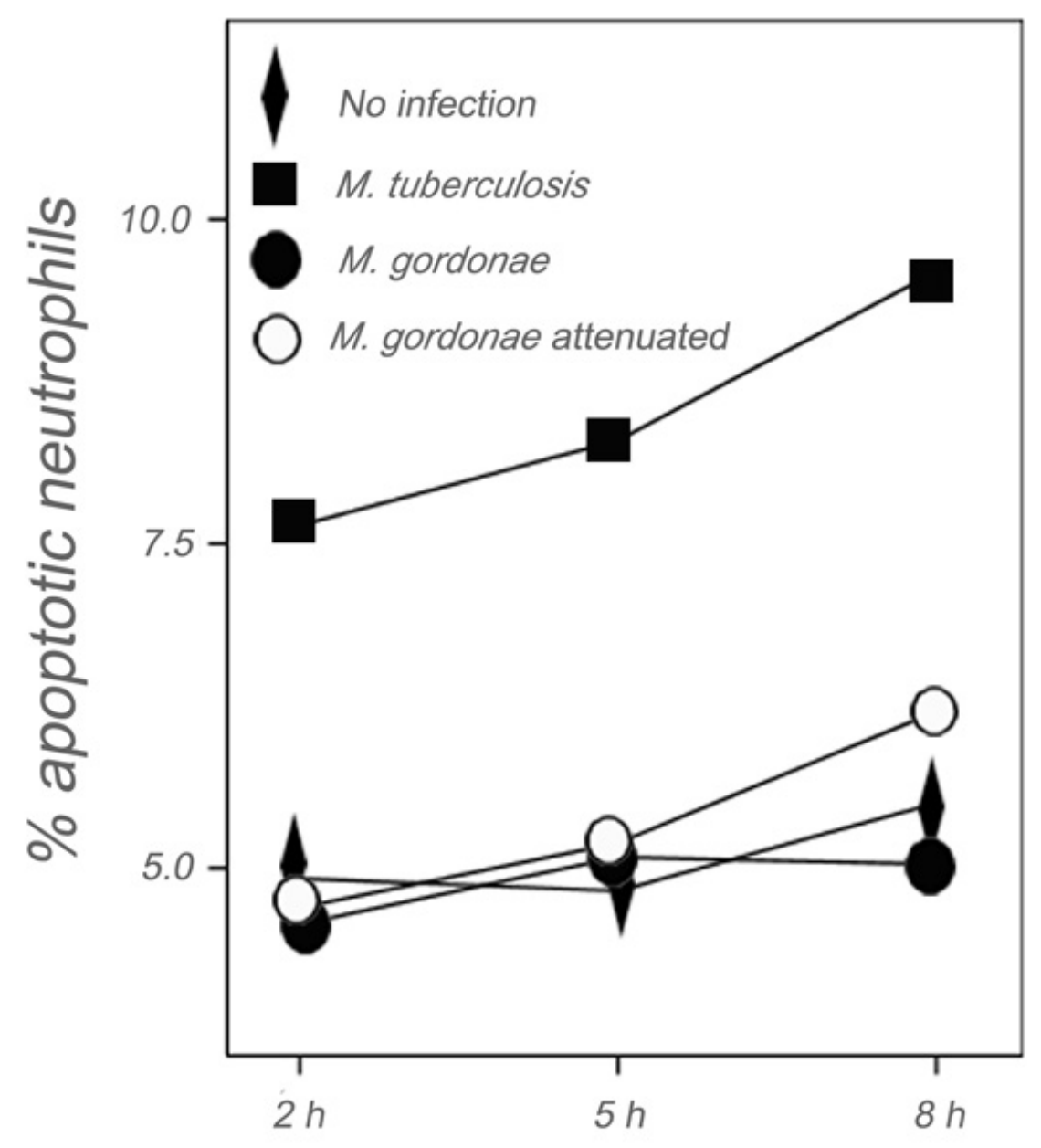

Figure 3. Apoptosis in infected neutrophils. $2 \times 10^{5}$ neutrophils were infected with $1 \times 10^{6}$ bacteria (M. tuberculosis, M. gordonae or M. gordonae attenuated). Non infected cells were used to measure spontaneous apoptosis. Data are the proportions (expressed as percentage) of fluorescein labelled cells (apoptotic) with respect to DAPI labelled cells (total) and represent the mean of four independent experiments, determined at 2, 5 and $8 \mathrm{~h}$. 


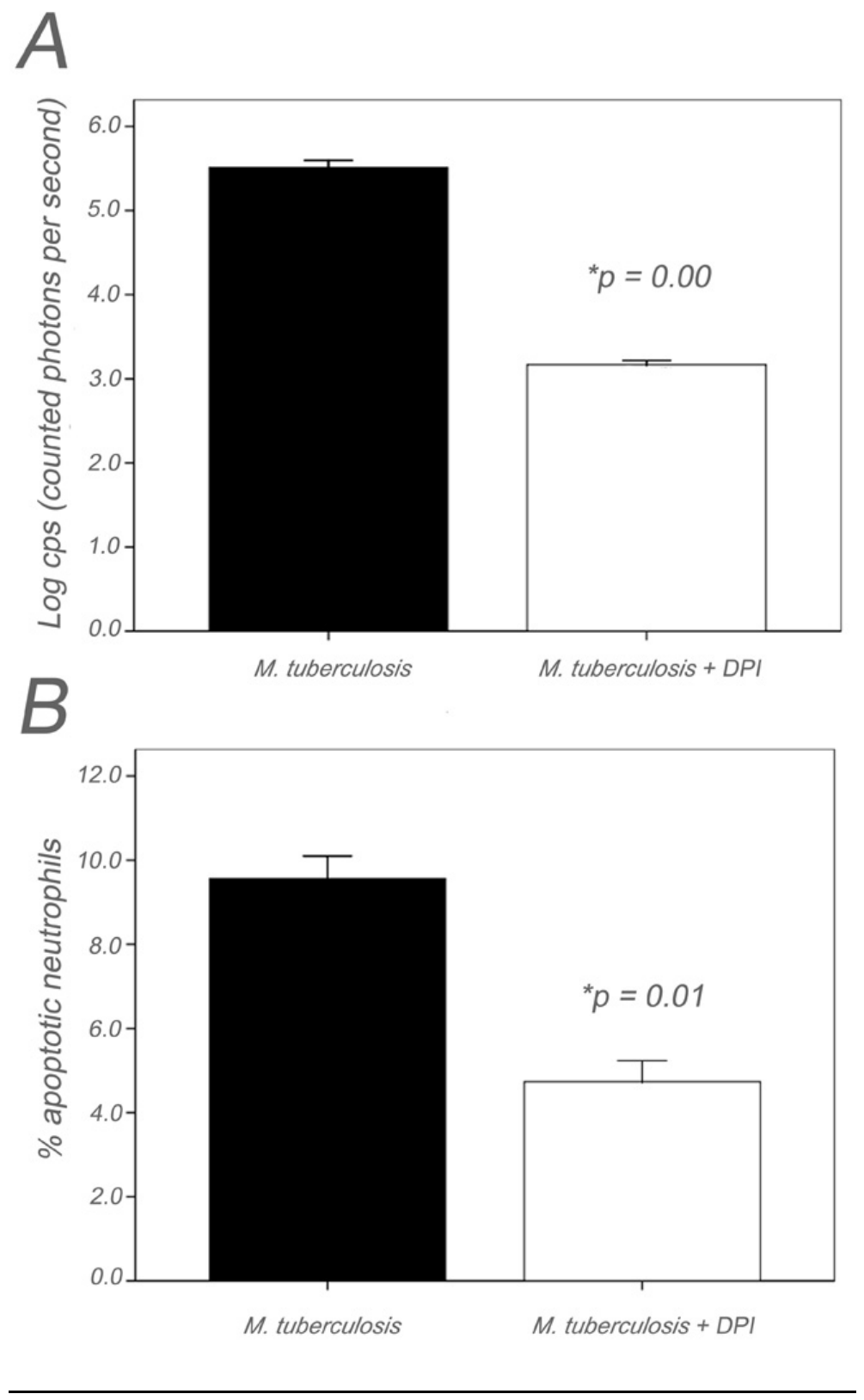

Figure 4. Influence of the NADPH oxidase inhibitor DPI in the ROS production and apoptosis of $M$. tuberculosis infected neutrophils. A) $4 \times 10^{5}$ neutrophils were infected with $2 \times 10^{6}$ bacteria. Data are the log of counted photons per second emitted in the presence of luminol. B) $2 \times 10^{5}$ neutrophils were infected with $1 \times 10^{6}$ bacteria. Data are the proportions (expressed as percentage) of fluorescein labelled cells (apoptotic) with respect to DAPI labelled cells (total). In both panels data represent the mean + S.D. of four independent experiment. Student $t$-test with $* p<0.05$ is considered significant 